\title{
Buttock Pain
}

National Cancer Institute

\section{Source}

National Cancer Institute. Buttock Pain. NCI Thesaurus. Code C78228.

Painful sensation in the buttocks. 